\title{
FROM PATHS TO STARS
}

\author{
A.F. ALAMEDDINE \\ Department of Mathematical Sciences \\ King Fahd University of Petroleum and Minerals \\ Dhahran 31261, Saudi Arabia \\ (Received March 14, 1990 and in revised form August 13, 1990)
}

\begin{abstract}
The number of cycles in the complement $T^{\prime}$ of a tree $T$ is known to increase with the diameter of the tree. A smimilar question is raised and settled for the number of complete subgraphs in $T^{\prime}$ for a special class of trees via Fibonacci numbers. A structural characterization of extremal trees is also presented.
\end{abstract}

KEY WORDS AND PHRASES. Cycles, Complete subgraphs, Trees. 1980 AMS SUBJECT CLASSIFICATION CODE. 05-C.

\section{INTRODUCTION.}

Among all trees $T$ of order $n$, the number $c\left(T^{\prime}\right)$ of all cycles in the complement $T^{\prime}$ and the structural characterization of those trees which optimize $c\left(T^{\prime}\right)$ have been dealt with in $[1,2]$. The same problem was solved in [3] for the number $i\left(T^{\prime}\right)$ of all complete subgraphs in the complement of an arbitrary $T$. It turns out that among all $n$-trees $T$, the path $P_{n}(n \geq 9)$ has both the maximum number of cycles [1] and the minimum number of complete subgraphs in its complement [3]. The star $S_{n}$ also maximizes both $c\left(T^{\prime}\right), 5 \leq n \leq 8[1]$ and $i\left(T^{\prime}\right)$ for $n \geq 4$ [2]. It minimizes $c\left(T^{\prime}\right)$ for $n \geq 9$.

The problem of characterizing the $n$-vertex trees $T$ for extremal values with respect to $c\left(T^{\prime}\right)$ or $i\left(T^{\prime}\right)$ loses some structural significance in the generality of $Y$. Suppose we consider a class of trees which keep out all paths and stars, for example, the class $\mathcal{F}_{3}$ of all those trees $T_{3}$ having exactly three endvertices. What structural similarities between $c\left(T_{3}^{\prime}\right)$ and $i\left(T_{3}^{\prime}\right)$ are inherited from $c\left(T^{\prime}\right)$ and $i\left(T^{\prime}\right)$ ? We recall that the diameter of a graph $G$ is the maximum distance $d(u, v)$ taken over all pairs of vertices $u, v$ in $G$. The following theorem $[1, \mathrm{p} .93]$ relates $c\left(T^{\prime}\right)$ with the diameter of $T$.

THEOREM 1. For each $n \geq 6$ and every tree $T$ of order $n$ and diameter $d, 4 \leq d \leq n-2$, there is a tree $T_{1}$ of order $n$ and at least diameter $d+1$ such that $c\left(T^{\prime}\right)<c\left(T_{1}^{\prime}\right)$.

\section{TREES WITH THREE ENDVERTICES.}

Utilizing enumerative techniques [2] we conclude that among all trees $T_{3}$ of order $n$, the 
tree with the smallest number of cycles in its complement is a tree with the smallest diameter as shown in Figure 1. Moreover, the tree $T_{3}$

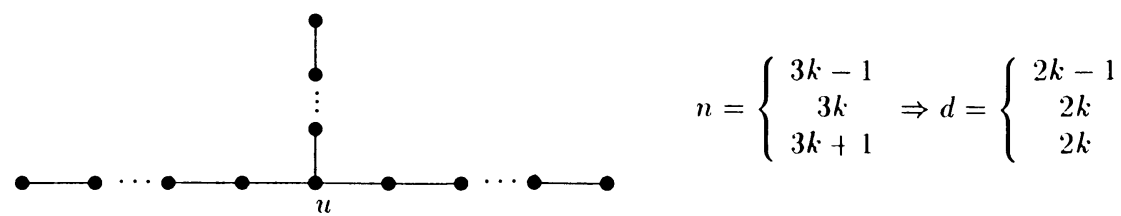

Figure 1. A tree with three endvertices and minimum $c\left(T_{3}^{\prime}\right)$.

with the largest number of cycles in its complement is a tree with the largest diameter as shown in Figure 2.

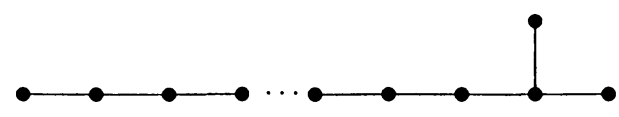

Figure 2. A trec with maximum $c\left(T_{3}^{\prime}\right)$.

So, among all trees with three endvertices, of order $n$, the direct relationship between $c\left(T_{3}^{\prime}\right)$ and the diameter of $T_{3}$ is inherited from the class of all trees, i.e., $\min _{\mathcal{F}_{3}} c\left(T_{3}^{\prime}\right)$ and $\max _{\mathcal{F}_{3}} c\left(T_{3}^{\prime}\right)$ are still associated with the smallest and the largest diameters of $T_{3}$, respectively. Can we make the same claim about $i\left(T_{3}^{\prime}\right)$, the number of complete subgraphs in the complement of $T_{3}$ ? We recall that when $T$ is arbitrary, $i\left(T^{\prime}\right)$ is maximum when the diameter of $T$ is minimum ( $T$ is a star) and $i\left(T^{\prime}\right)$ is minimum when the diameter is maximum ( $T$ is a path). Does this relationship between $i\left(T^{\prime}\right)$ and the diameter of $T$ remain true when $T$ is restricted to $\mathcal{F}_{3}$ ? To this end, we need the concept of a Fibonacci number $f(G)$ of a graph $G$.

According to $[4$, p. 45], the total number of subsets of $\{1,2,3, \ldots, n\}$ such that no two elements are adjacent is $F_{n+1}$, where $F_{n}$ is the $n$th Fibonacci number, which is defined by

$$
F_{0}=F_{1}=1, F_{n}=F_{n-1}+F_{n-2}, \quad n \geq 2 .
$$

The sequence $\{1,2,3, \ldots, n\}$ can be regarded as the vertex set of the path $P_{n}$. This definition covers the empty graph also; so, $f(G)=i\left(G^{\prime}\right)$. We note that $i\left(P_{0}^{\prime}\right)=1, i\left(P_{1}^{\prime}\right)=$ $2, i\left(P_{2}^{\prime}\right), \ldots, i\left(P_{n}^{\prime}\right)=F_{n+1}$.

\section{MAIN RESULTS}

If $T_{3}$ is a tree with three endvertices, then it has a unique vertex $u$ of degree threc. We count $i\left(T_{3}^{\prime}\right)$ [5] by considering two disjoint sets of complete subgraphs of $T_{3}^{\prime}$, say $S_{1}$ and $S_{2}$, where $S_{1}$ is the set of those complete subgraphs not containing the vertex $u$, and $S_{2}$ consists of those that do contain $u$. Let $v_{1}, v_{2}$ and $v_{3}$ be the three vertices adjacent to $u$ in $T_{3}$. We have

$$
i\left(T_{3}^{\prime}\right)=\left|S_{1}\right|+\left|S_{2}\right|=i\left(T_{3}-u\right)^{\prime}+i\left(T_{3}-v_{1}-v_{2}-v_{3}\right)^{\prime} .
$$

If $n=3 k+1, T_{3}-u$ is a union of three disjoint paths on $3 k$ vertices, where $T_{3}-v_{1}-v_{2}-v_{3}$ 
is also a union of three disjoint paths on $3 k-3$ vertices together with the isolated vertex $u$ (see Figure 1). The following theorem on Fibonacci numbers shows that $i\left(T_{3}^{\prime}\right)$ is minimized and maximized by the trees in Figures $3 \mathrm{a}$ and $3 \mathrm{~b}$, respectively. This shows

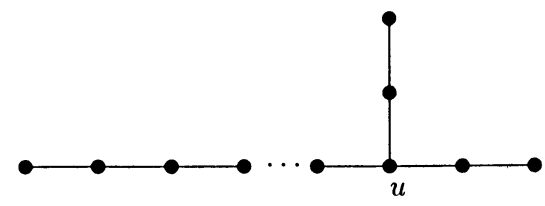

(a) $\min i\left(T_{3}^{\prime}\right)$

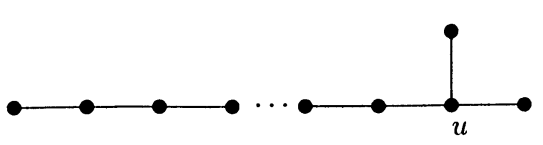

(b) $\max i\left(T_{3}^{\prime}\right)$

Figure 3. Extremal trees in $\mathcal{F}_{3}$.

that the inverse relationship between $i\left(T^{\prime}\right)$ and the diameter of $T$ is not inherited in the class of trees $\mathcal{F}_{3}$ with exactly three endvertices.

THEOREM 2. Let $n$ be an integer $\geq 7$. Then among all summands $r_{1}, s_{1}, t_{1}$ and $r_{2}, s_{2}, t_{2}$ satisfying (i) $r_{1}+s_{1}+t_{1}=n+2$, (ii) $r_{1}=r_{2}+1, s_{1}=s_{2}+1, t_{1}=t_{2}+1$, and $r_{1}, s_{1}, t_{1} \geq 2$ we have

the sum of the two products $F_{r_{1}} F_{s_{1}} F_{t_{1}}+F_{r_{2}} F_{s_{2}} F_{t_{2}}$ of the Fibonacci numbers $F_{r_{1}}, F_{s_{1}}, F_{t_{1}}$ and $F_{r_{2}}, F_{s_{2}}, F_{t_{2}}$ is

(a) minimum if $r_{1}=s_{1}=3$ and $t_{1}=n-4$ and

(b) maximum if $r_{1}=s_{1}=2$ and $t_{1}=n-2$.

PROOF. The order of growth of $F_{n}$ is governed by the golden ratio $\tau=(1+\sqrt{5}) / 2$. Moreover, $F_{n} \approx c \tau^{n}$ where $c=\tau / \sqrt{5}$ and $F_{n+1} \approx \tau F_{n}$. We have $r_{1}+s_{1}+t_{1}=n+2$ and $r_{2}+s_{2}+t_{2}=n-1$, and for large $n$,

$$
\begin{aligned}
F_{r_{1}} F_{s_{1}} F_{t_{1}} & +F_{r_{2}} F_{s_{2}} F_{t_{2}} \approx\left(\tau F_{r_{2}}\right)\left(\tau F_{s_{2}}\right)\left(\tau F_{t_{2}}\right)+F_{r_{2}} F_{s_{2}} F_{t_{2}}=\left(1+\tau^{3}\right) F_{r_{2}} F_{s_{2}} F_{t_{2}} \\
& \approx(5.236068 \cdots)\left(c^{3} \tau^{r_{2}+s_{2}+t_{2}}\right) \approx 13.59764677 \cdots \tau^{n-5}>13.43181071 \cdots \tau^{n-5} \\
& \approx 9 F_{n-4}+4 F_{n-5}=F_{3} F_{3} F_{n-4}+F_{2} F_{2} F_{n-5} \\
& =i\left(P_{2}^{\prime}\right) i\left(P_{2}^{\prime}\right) i\left(P_{n-5}^{\prime}\right)+i\left(P_{1}^{\prime}\right) i\left(P_{1}^{\prime}\right) i\left(P_{n-6}^{\prime}\right) .
\end{aligned}
$$

That is, $\min _{\mathcal{F}_{3}} i\left(T_{3}^{\prime}\right)$ is realized in Figure 3a.

To prove (b), we note that for the tree in Figure $3 \mathrm{~b}$, we have $i\left(T_{3}^{\prime}\right)=4 F_{n-2}+F_{n-3} \approx$ $12.09016992 \cdots F_{n-4}$. On the other hand, for an arbitrary $T_{3}$ with $n$ large enough, we have $F_{r_{1}} F_{s_{1}} F_{t_{1}}+F_{r_{2}} F_{s_{2}} F_{t_{2}} \approx 13.59764677 \cdots \tau^{n-5} \approx 11.61377685 \cdots F_{n-4}<12.09016992 \cdots F_{n-4} \approx$ $4 F_{n-2}+F_{n-3}$. That is, $\max _{\mathcal{F}_{3}} i\left(T_{3}^{\prime}\right)$ is realized in Figure $3 \mathrm{~b}$.

ACKNOWLEDGEMENTS. The author gratefully acknowledges the support of King Fahd University of Petroleum and Minerals and wishes to thank the anonymous referee for his valuable hints. 


\section{REFERENCES}

1. ZIIOU, B. The maximum number of cycles in the complement of a tree, Discrete Math. 69(1988), No. 1, 85-94.

2. REID, K.B. Cycles in the complement of a tree, Discrete Math. 15(1976) 163-174.

3. PRODINGER, H., TICHY, R.F. Fibonacci numbers of graphs, The Fibonacci Quarterly, 20(1982), p. 16.

4. COMTET, L. Advanced Combinatorics, Dordrecht, Holland: Reidel, 1974.

5. ALAMEDDINE, A.F. Fibonacci numbers and bipyramids, The Fibonacci Quarterly, $\underline{27}(1989), 247-252$. 


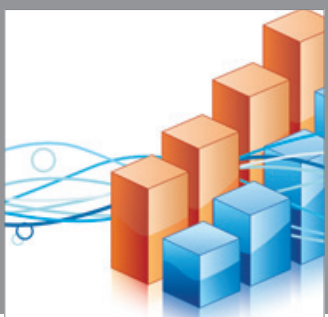

Advances in

Operations Research

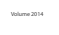

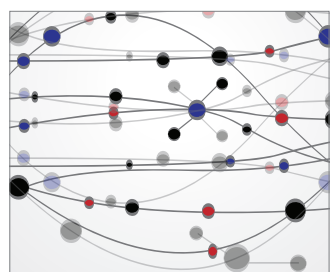

\section{The Scientific} World Journal
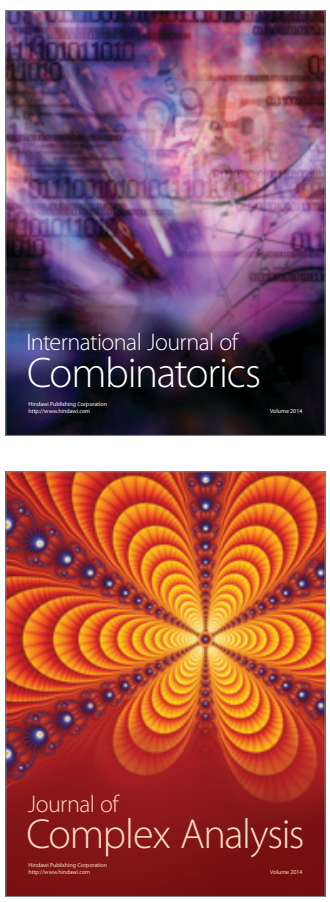

International Journal of

Mathematics and

Mathematical

Sciences
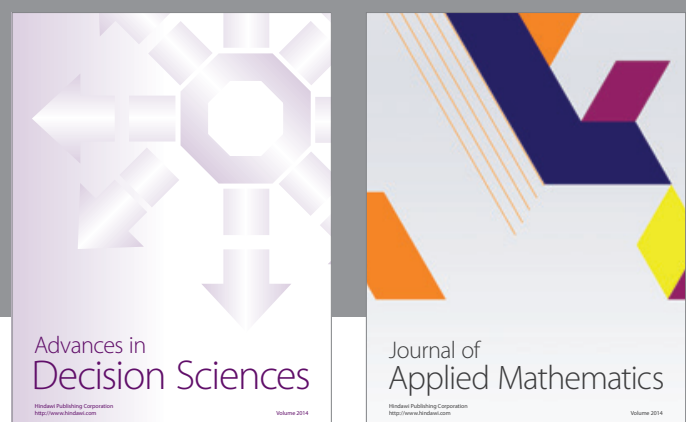

Journal of

Applied Mathematics
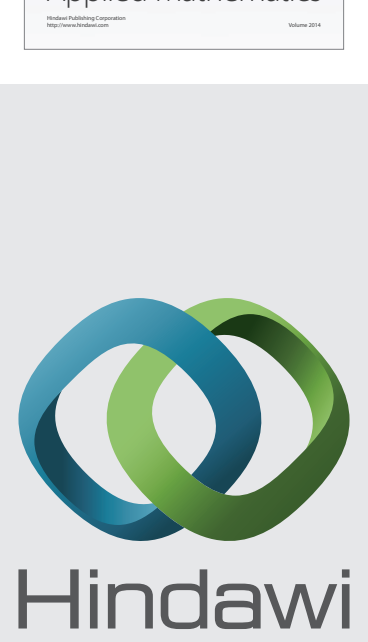

Submit your manuscripts at http://www.hindawi.com
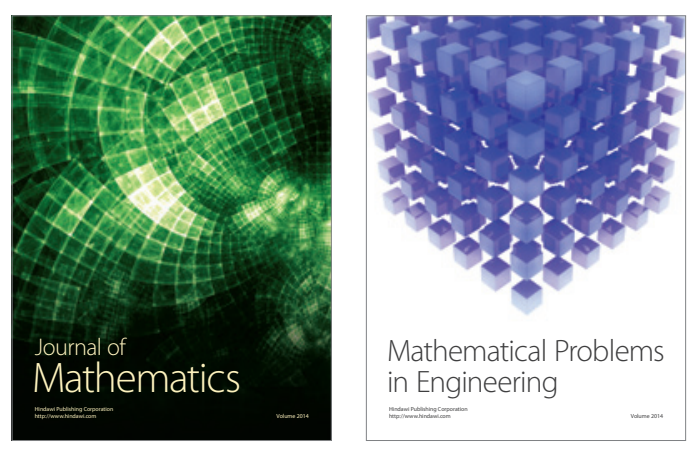

Mathematical Problems in Engineering
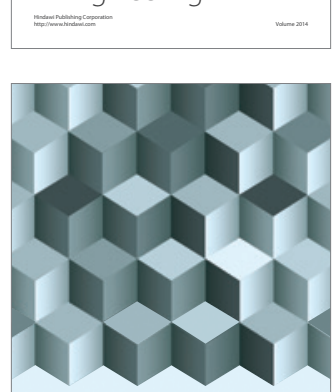

Journal of

Function Spaces
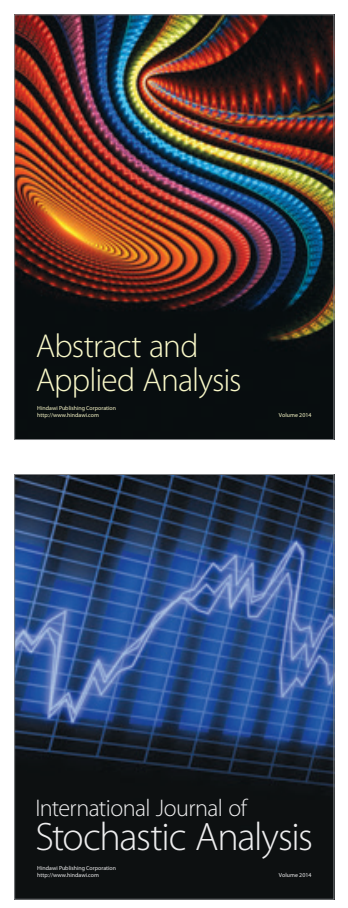

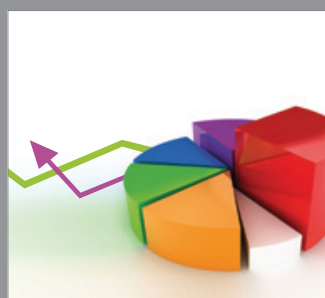

ournal of

Probability and Statistics

Promensencen
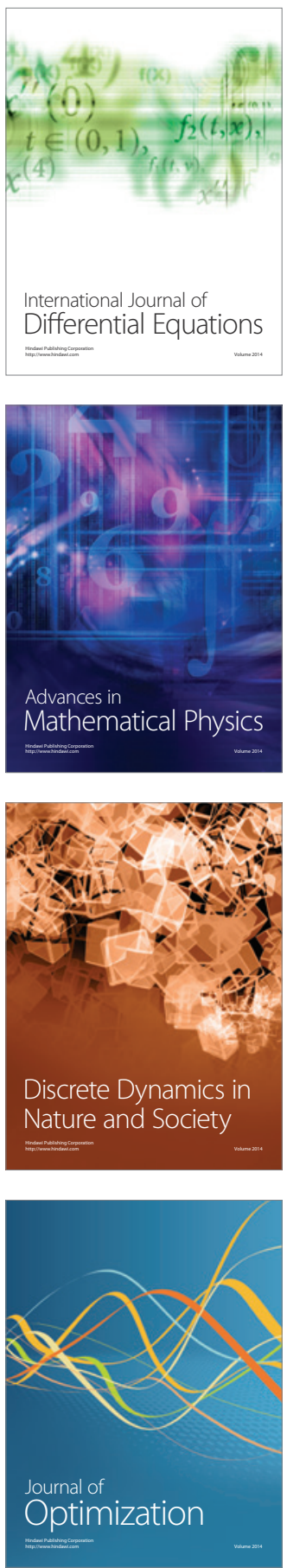